\title{
L'influence des matchs de hockey professionnel vus à la télévision sur les blessures des jeunes hockeyeurs
}

\author{
G. Keays, M. Sc. (1); B. Pless, M.D. (2)
}

Cet article a fait l'objet d'une évaluation par les pairs.

\section{Résumé}

Introduction : La plupart des matchs de la Ligue nationale de hockey (LNH) télédiffusés comportent des mises en échec violentes, des coups illégaux et des altercations. Nous avons émis l'hypothèse que les joueurs des ligues de hockey mineur imitaient de tels comportements et que le fait de ne pas regarder ces matchs se traduirait par une réduction du taux de blessures chez les joueurs de hockey plus jeunes.

Méthodologie : En utilisant un modèle quasi expérimental, nous avons comparé sept ans de matchs de la LNH télédiffusés (2002-2009) avec l'année du lock-out de la LNH (2004-2005). Nous avons utilisé les données du Système canadien hospitalier d'information et de recherche en prévention des traumatismes (SCHIRPT) pour caractériser les blessures et déterminer si elles étaient attribuables à un contact intentionnel ou à des actes illégaux, ce qui inclut les altercations.

Résultats : Nous n’avons observé aucune différence significative entre les proportions de blessures tous types confondus et celles liées à un contact intentionnel, un acte violent ou un acte illégal chez les joueurs de sexe masculin des ligues de hockey mineur entre l'année où les joueurs professionnels ont été en lock-out et les années précédentes et suivantes.

Conclusion : Nous concluons que le fait de ne pas voir de matchs télédiffusés de la LNH comportant des scènes violentes pourrait ne pas se traduire par une réduction des blessures, même si un effet possible pourrait avoir été masqué puisque nous avons observé une augmentation marquée, durant le lock-out, du nombre de spectateurs aux matchs des ligues mineures professionnelles où la violence est tout aussi présente.

Mots-clés : adolescents, garçons, écoute de la télévision, violence, blessures sportives, hockey

\section{Introduction}

" Bien sûr qu'on essaie de faire comme eux. On les voit faire toutes sortes de choses sans jamais être punis. " Ainsi s'exprimait un jeune joueur de hockey de 12 ans interviewé à la télévision canadienne après la mise en échec dans un angle mort subie par la vedette de la Ligue nationale de hockey (LNH) Sidney Crosby, ce qui lui a valu une commotion cérébrale l'obligeant à rester à l'écart du jeu pendant près de onze mois. Le décès récent de plusieurs «bagarreurs » de la LNH, c'est-àdire de joueurs dont le rôle principal est justement de se bagarrer, est venu alimenter le débat sur la violence dans le hockey.

L'influence des médias sur le comportement des téléspectateurs est au centre de controverses depuis les années 1950 ${ }^{1-3}$. Ainsi, la question de savoir si le fait, pour les enfants, de voir des scènes violentes à la télévision a un effet négatif sur eux n'a toujours pas été tranchée. En 1975, Rothenberg a résumé 146 études en concluant que « la violence à l'écran avait pour effet de rendre les jeunes téléspectateurs plus agressifs ${ }^{4}$. Toutefois, des rapports plus récents, dont plusieurs examens systématiques et méta-analyses, ont donné lieu à des conclusions divergentes, allant de l'absence d'effet ${ }^{5}$ à une nocivité avérée $e^{6-11}$. Néanmoins, pour l'American Psychological Association ${ }^{12}$ et l'American Academy of Pediatrics ${ }^{13}$, la prépondérance de la preuve amène à conclure à des effets négatifs.

Si les scènes violentes à l'écran vues par les enfants prennent principalement la forme de dessins animés ou de films d'action, elles sont également très fréquentes dans de nombreuses émissions sportives. Le hockey sur glace, en particulier, a la réputation de faire autant appel à l'agression qu'à l'habileté du jeu. Cette discipline est à l'origine du taux le plus élevé de blessures sportives chez les garçons $^{14}$ et n'est devancée que par le football dans les premières causes de lésions médullaires graves ${ }^{15}$. Le degré de violence présent dans les matchs de hockey télédiffusés est saisissant : environ $40 \%$ des matchs de la LNH incluent au moins une altercation ${ }^{16}$ et quelque $16 \%$ de toutes les blessures graves (c.-à-d. celles qui contraignent un joueur à quitter le jeu) ont pour origine des comportements ayant donné lieu à une pénalité ou à une suspension $^{17}$. Dans les ligues de hockey mineur professionnel, considérées par plusieurs comme les plus violentes de ce sport, il est habituel d'assister à trois ou

Rattachement des auteurs :

1. Centre universitaire de santé McGill, Hôpital de Montréal pour enfants, Montréal (Québec), Canada

2. Départements de pédiatrie et d'épidémiologie et biostatistiques, Université McGill, Montréal (Québec), Canada

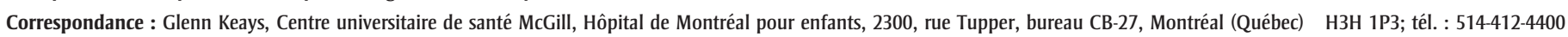
poste 23167; téléc. : 514412-4477; courriel : glenn@keays.ca 
quatre altercations par partie ${ }^{18}$. La mise en échec par l'arrière, une manœuvre généralement associée à des blessures graves, n'est devenue illégale qu'en $2000^{19}$, alors que les coups délibérés visant la tête (« coups à la tête ») suscitent toujours la controverse $^{20}$. À cause du caractère " macho » du hockey professionnel, le port du casque n'a été introduit qu'en $1979^{21}$ et l'utilisation obligatoire de la visière continue d'être reportée ${ }^{22}$. Au hockey mineur, ces deux pièces d'équipement sont obligatoires depuis de nombreuses années.

Le comportement des enfants et des jeunes jouant dans les ligues de hockey mineur semble être influencé par les parties de la LNH qu'ils regardent à la télévision ${ }^{23-27}$. Une enquête a montré que $90 \%$ des jeunes interrogés ont affirmé avoir appris " un comportement, une technique ou une habileté » en regardant les joueurs de hockey professionnels. De plus, $56 \%$ ont déclaré avoir copié des tactiques de joueurs professionnels illégales au moins une fois pendant la saison de hockey en $\operatorname{cours}^{28}$. Une autre enquête a révélé que les joueurs de hockey des écoles secondaires qui avaient pour modèles des joueurs agressifs de la LNH étaient plus nombreux à commettre des agressions à l'égard d'autres joueurs pendant un match ${ }^{29}$. Plus récemment, dans un rapport commandé par le ministère des Sports de la Colombie-Britannique, on a noté que $27 \%$ des 144 jeunes joueurs de hockey ayant participé à l'enquête avaient imité des coups illégaux portés par des joueurs de la LNH vus à la télévision ${ }^{30}$.

Nous en avons conclu que nous pouvions raisonnablement formuler l'hypothèse selon laquelle le fait de ne pas regarder le hockey professionnel à la télévision était de nature à améliorer le comportement des joueurs plus jeunes, de sorte qu'il y ait moins de blessures. Pour mettre cette hypothèse à l'épreuve, nous avons tiré parti d'une expérience qui s'est déroulée dans des conditions naturelles : au cours de l'hiver 2004-2005, les propriétaires des équipes ont imposé un lockout aux joueurs de la LNH durant un litige contractuel. En conséquence, exception faite des rediffusions d'anciens matchs de la LNH en avril 2005 et des matchs de championnat des ligues juniors à la fin du mois de mai, il n’y a pas eu de hockey à la télévision canadienne. Nous avons examiné si l'absence de hockey professionnel télévisé durant cette saison avait été associée à un taux de blessures moins élevé chez les joueurs des ligues mineures.

\section{Méthodologie}

Notre étude a porté exclusivement sur les garçons ayant joué au hockey dans des ligues de hockey mineur au Canada au cours de sept saisons successives, la première étant celle de 2002-2003. Les ligues de hockey mineur sont subdivisées en différents niveaux en fonction de l'âge des joueurs, à savoir Peewee, Bantam et Midget $^{31}$.

Nous n'avons pris en considération que les blessures survenues durant la saison régulière de la LNH. Nous avons obtenu des renseignements détaillés concernant les blessures dans le Système canadien hospitalier d'information et de recherche en prévention des traumatismes

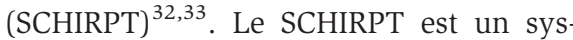
tème de surveillance des blessures qui a été mis en œuvre dans 14 services hospitaliers d'urgence de sept provinces. Il recueille de l'information auprès des parents des patients (ou des patients s'ils sont plus âgés) au sujet des circonstances dans lesquelles la blessure est survenue, et contient des renseignements médicaux détaillés tels que la nature de la blessure, la partie du corps atteinte et le traitement dispensé.

Nous avons utilisé plusieurs définitions pour décrire la cause ou le mécanisme de la blessure. Au départ, nous avons comparé toutes les blessures aux «blessures liées à un contact», ce qui inclut tous les types de contact, qu'ils soient intentionnels ou pas. Ensuite, nous avons analysé deux types particuliers de contact. Le premier, les «blessures causées par un contact illégal », fait référence aux cas causés par un coup (ou un acte) illégal au sens de Hockey Canada ${ }^{31}$. Appartiennent à cette catégorie les actes suivants : coup de coude (lever le coude de manière à causer une blessure), double échec (le fait de se servir de la partie du bâton située entre les deux mains pour arrêter un adversaire par la force), mise en échec par derrière, plaquage contre la bande (mise en échec d'un adversaire sans défense de manière à le projeter violemment sur la bande), mise en échec à la tête, coup de genou (avancer le genou pour provoquer un contact avec l'adversaire), cinglage (action de frapper avec force un adversaire avec son bâton), faire trébucher (placer le bâton, le genou, le pied, le bras, la main ou le coude de manière à faire trébucher ou tomber l'adversaire), rudesse, ou tout acte de violence tel que bataille, altercation et coups de poing délibérés. La seconde catégorie, "blessures causées par des altercations ", inclut toutes les blessures découlant de batailles, altercations et coups de poing délibérés.

Pour calculer les taux, nous avons obtenu de Hockey Canada, pour chaque année de l'étude, le nombre de garçons âgés de 11 à 17 ans inscrits dans chacune des ligues de hockey mineur et nous avons exprimé la proportion en nombre de blessures pour 1000 joueurs de sexe masculin inscrits dans toutes les villes possédant un hôpital pédiatrique partenaire du SCHIRPT. Les intervalles de confiance des taux individuels et des proportions individuelles ont été calculés à l'aide du test de Poisson.

\section{Résultats}

Entre les mois de septembre et d'avril des années 2002 à 2009, le SCHIRPT fait état de 14717 blessures au hockey chez les garçons de 11 à 17 ans. Parmi les blessés, $24 \%$ étaient de niveau Peewee (11 à 12 ans), 39\%, de niveau Bantam (13 à 14 ans) et $37 \%$, de niveau Midget (15 à 17 ans). Pour la plupart des années, à chaque niveau, environ $70 \%$ des blessures étaient liées à un contact. Pour tous les niveaux d'âge combinés, les taux pour 1000 joueurs inscrits variaient entre 19,0 et 24,9 pour une blessure en général et entre 13,7 et 18,4 pour une blessure considérée comme liée à un contact (tableau 1). Toutefois, les données ne font ressortir aucune tendance au fil du temps et aucune preuve d'un changement marqué de la proportion de blessures durant l'année du lock-out par rapport aux années précédentes ou suivantes. Il en est de même lorsqu'on examine ces données par ligue ou par groupe d’âge. 
TABLEAU 1

Taux approximatifs ${ }^{\mathrm{a}}$ de blessures au hockey, tous types de blessures et blessures liées au contact, par ligue (groupe d'âge) et par saison pour 1000 joueurs des ligues de hockey mineur (11-17 ans), tous les centres SCHIRPT, Canada

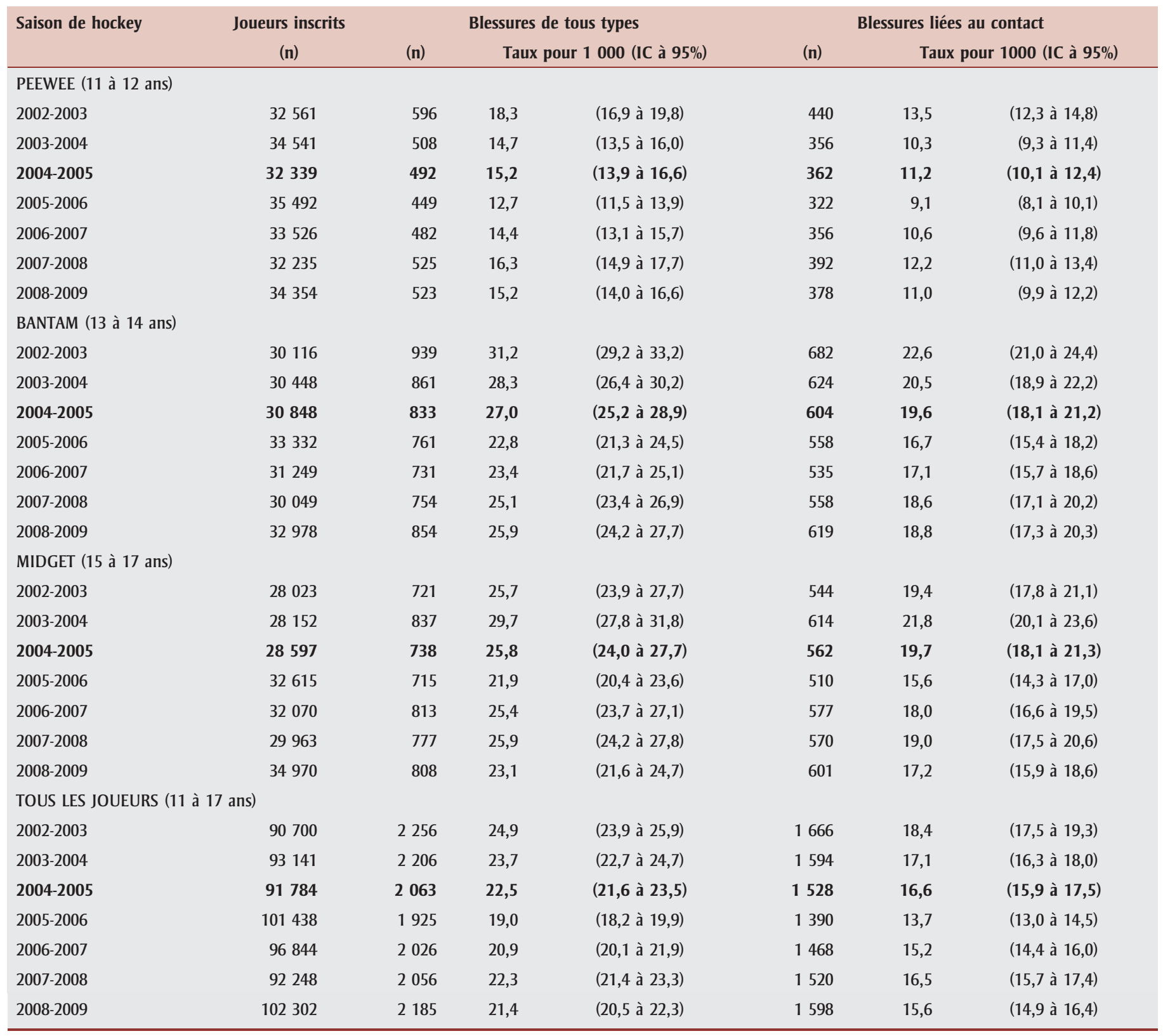

Sources : SCHIRPT ${ }^{32}$; Hockey Canada (http://www.hockeycanada.ca/index.php/ci_id/23952/la_id/2.htm.

Abréviations : LNH, Ligne nationale de hockey; SCHIRPT, Système canadien hospitalier d'information et de recherche en prévention des traumatismes.

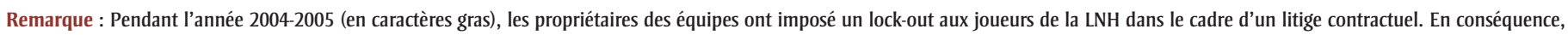

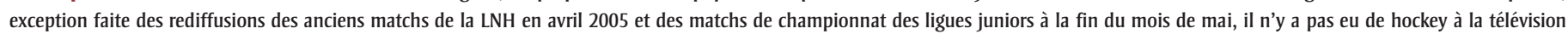
canadienne.

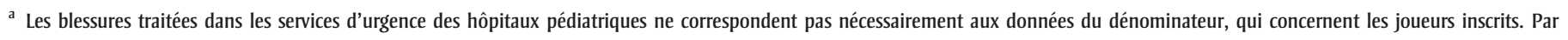
conséquent, les taux que nous avons utilisés ne sont pas de «véritables » taux, car les données des numérateurs et des dénominateurs proviennent de populations différentes.

Même si les différences ne sont pas statistiquement significatives, le tableau 2 indique une tendance constante en faveur d'un nombre légèrement supérieur de blessures découlant d'actes qui ont été jugés dangereux, c'est-à-dire intentionnels ou illégaux, durant l'année du lock-out.
La figure 1 montre le nombre de spectateurs des matchs des ligues de hockey mineur professionnel avant, pendant et après le lock-out. Nous pensions que, privés de matchs de la LNH à la télévision, les amateurs fervents compenseraient en regardant ces matchs, dont certains étaient télédiffusés. Cette figure indique clairement un pic du nombre de spectateurs à ces matchs durant le lockout; ce que la figure ne révèle cependant pas, c'est que de l'avis de nombreuses personnes, les spectateurs regardent ces matchs en partie à cause de leur caractère 
TABLEAU 2

Proportions des blessures attribuables à des actes illégaux et à des altercations durant les matchs de hockey organisés, par ligue de hockey mineur et par année, 2002-2003 à 20082009

\begin{tabular}{|c|c|c|c|c|c|}
\hline \multirow[t]{2}{*}{ Saison de hockey } & \multirow{2}{*}{$\begin{array}{c}\text { Toutes les } \\
\text { blessures } \\
\text { (n) }\end{array}$} & \multicolumn{2}{|c|}{$\begin{array}{c}\text { Blessures attribuables à des } \\
\text { actes illégáaux }\end{array}$} & \multicolumn{2}{|c|}{$\begin{array}{c}\text { Blessures attribuables à des } \\
\text { altercations }\end{array}$} \\
\hline & & $\%$ & (IC) & $\%$ & (IC) \\
\hline \multicolumn{6}{|c|}{ PEEWEE (11 à 12 ans) } \\
\hline $2002-2003$ & 596 & 22,5 & $(18,1$ à 26,9$)$ & 0,5 & $(0,0$ à 1,3$)$ \\
\hline 2003-2004 & 508 & 16,7 & $(12,5$ à 21,0$)$ & 0,2 & $(0,0$ à 0,8$)$ \\
\hline 2004-2005 & 492 & 27,4 & $(22,3$ à 32,7$)$ & 1,2 & $(0,0$ à 2,5$)$ \\
\hline 2005-2006 & 449 & 25,4 & $(20,1$ à 30,7$)$ & 0,4 & $(0,0$ à 1,3$)$ \\
\hline 2006-2007 & 482 & 21,8 & $(17,0$ à 26,7$)$ & 0,2 & $(0,0$ à 0,8$)$ \\
\hline 2007-2008 & 525 & 26,5 & $(21,6$ à 31,5$)$ & 0,6 & $(0,0$ à 1,5$)$ \\
\hline 2008-2009 & 523 & 22,9 & (18,3 à 27,7$)$ & 0,2 & $(0,4$ à 0,7$)$ \\
\hline \multicolumn{6}{|c|}{ BANTAM (13 à 14 ans) } \\
\hline $2002-2003$ & 939 & 17,1 & $(14,0$ à 20,4$)$ & 0,2 & $(0,0$ à 0,7$)$ \\
\hline 2003-2004 & 861 & 13,8 & $(10,8$ à 16,9$)$ & 0,6 & $(0,0$ à 1,3$)$ \\
\hline 2004-2005 & 833 & 18,7 & $(15,3$ à 22,3$)$ & 1,0 & $(0,1$ à 1,9$)$ \\
\hline 2005-2006 & 761 & 18,3 & $(14,7$ à 21,9$)$ & 0,4 & $(0,0$ à 1,0$)$ \\
\hline 2006-2007 & 731 & 18,5 & $(14,8$ à 22,2$)$ & 0,8 & $(0,0$ à 1,7$)$ \\
\hline 2007-2008 & 754 & 16,4 & $(13,0$ à 20,0$)$ & 0,9 & $(0,1$ à 1,9$)$ \\
\hline 2008-2009 & 854 & 17,6 & $(14,3$ à 21,0$)$ & 0,5 & $(0,0$ à 1,1$)$ \\
\hline \multicolumn{6}{|c|}{ MDGET (15 à 17 ans) } \\
\hline $2002-2003$ & 721 & 17,2 & $(13,6$ à 20,9$)$ & 1,9 & $(0,7$ à 3,3$)$ \\
\hline 2003-2004 & 837 & 19,5 & $(16,0$ à 23,1$)$ & 1,9 & $(0,7$ à 3,2) \\
\hline 2004-2005 & 738 & 23,2 & $(19,2$ à 27,2$)$ & 2,7 & $(1,2$ à 4,3$)$ \\
\hline 2005-2006 & 715 & 19,3 & $(15,5$ à 23,2$)$ & 1,3 & $(0,2$ à 2,4$)$ \\
\hline 2006-2007 & 813 & 17,2 & $(13,9$ à 20,7$)$ & 1,6 & $(0,5$ à 2,8$)$ \\
\hline 2007-2008 & 777 & 19,9 & (16,3 à 23,7$)$ & 2,1 & $(0,8$ à 3,4) \\
\hline 2008-2009 & 808 & 19,3 & $(15,8$ à 22,9$)$ & 1,6 & $(0,5$ à 2,8$)$ \\
\hline \multicolumn{6}{|c|}{ TOUS LES JOUEURS (11 à 17 ans) } \\
\hline $2002-2003$ & 2256 & 18,6 & (16,5 à 20,7) & 0,8 & $(0,4$ à 1,4$)$ \\
\hline 2003-2004 & 2206 & 16,6 & (14,6 à 18,7) & 1,0 & $(0,5$ à 1,6) \\
\hline 2004-2005 & 2063 & 22,4 & (20,1 à 24,8) & 1,6 & $(1,0$ à 2,4$)$ \\
\hline 2005-2006 & 1925 & 20,3 & $(18,0$ à 22,7$)$ & 0,7 & $(0,3$ à 1,3$)$ \\
\hline 2006-2007 & 2026 & 18,8 & $(16,6$ à 21,0$)$ & 1,0 & $(0,5$ à 1,6) \\
\hline 2007-2008 & 2056 & 20,3 & (18,1 à 22,7) & 1,3 & (0,7 à 1,9) \\
\hline 2008-2009 & 2185 & 19,5 & $(17,4$ à 21,7$)$ & 0,8 & $(0,4$ à 1,4$)$ \\
\hline
\end{tabular}

Source : Système canadien hospitalier d'information et de recherche en prévention des traumatismes ${ }^{32}$; Hockey Canada (http://www.hockeycanada.ca/index.php/ci_id/23952/la_id/2.htm)

Abréviation : LNH, Ligue nationale de hockey.

Remarque : Pendant l'année 2004-2005 (en caractères gras), les propriétaires des équipes ont imposé un lock-out aux joueurs de la LNH dans le cadre d'un litige contractuel. En conséquence, exception faite des rediffusions des anciens matchs de la LNH en avril 2005 et des matchs de championnat des ligues juniors à la fin du mois de mai, il n’y a pas eu de hockey à la télévision canadienne.

a Actes illégaux : accrochage, faire trébucher, retenir le bâton, double échec, mise en échec par derrière, cinglage, donner du coude, placage sur la bande, mise en échec à la tête, coup de genou et rudesse.

b Batailles et altercations.

violent $^{34,35}$. Les joueurs et les entraîneurs de ces équipes acceptent que les « fiers-àbras » (hockeyeurs mettant l'accent sur l'intimidation et la violence) fassent matchs ${ }^{36,37}$. partie de l'attrait exercé par les

\section{Analyse}

Le hockey professionnel est violent parce qu'il repose sur un jeu agressif. Dans Violence and Sport, $\mathrm{Smith}^{28}$ définit l'agression comme étant " tout comportement ayant pour but de blesser psychologiquement ou physiquement une autre personne ". C'est une violence physique qui caractérise l'essentiel du hockey professionnel. Robidoux et Trudel $^{38}$ notent que "la mise en échec est un exemple de l'utilisation réglementée de la force physique pour obtenir un avantage [...] [et] mène sans conteste à une augmentation des blessures ". D'après plusieurs études antérieures, le fait pour les jeunes hockeyeurs d'observer le comportement des joueurs professionnels durant les matchs de hockey télévisés influence leur comportement ${ }^{25,28-30,39,40}$. Toutefois, contrairement à notre hypothèse de départ, nous n'avons relevé aucune différence systématique dans les taux de blessures de tous les types entre les saisons où les jeunes hockeyeurs ne regardaient pas les matchs de la LNH à la télévision et celles où ils les regardaient. Néanmoins, il demeure vraisemblable que les jeunes joueurs imitent les comportements violents qu'ils voient à la télévision, ce qui nous a amenés à essayer de d'interpréter nos résultats.

Une explication est que les comportements liés aux blessures chez les jeunes hockeyeurs sont si profondément ancrés que leur modification est peu probable après seulement une année passée sans le renforcement lié au fait de voir les joueurs professionnels en action. Une deuxième explication possible est que pour compenser, durant le lock-out, les jeunes joueurs ont assisté à un plus grand nombre de matchs des ligues de hockey mineur professionnel. Paradoxalement peut-être, ces matchs sont considérés comme étant plus violents que ceux de la $\mathrm{LNH}^{34-37}$, et il est intéressant de souligner, comme le montre la figure 1 , que le nombre de spectateurs à ces matchs a augmenté de manière frappante durant le lock-out ${ }^{41,42}$. Ainsi, l'exposition à la violence pourrait être restée la même pendant toute la période couverte par l'étude. 
FIGURE 1

Nombre de spectateurs aux matchs de deux ligues de hockey mineur professionnel (North American Hockey League et American Hockey League) entre les saisons de hockey 2002-2003 et 2008-2009

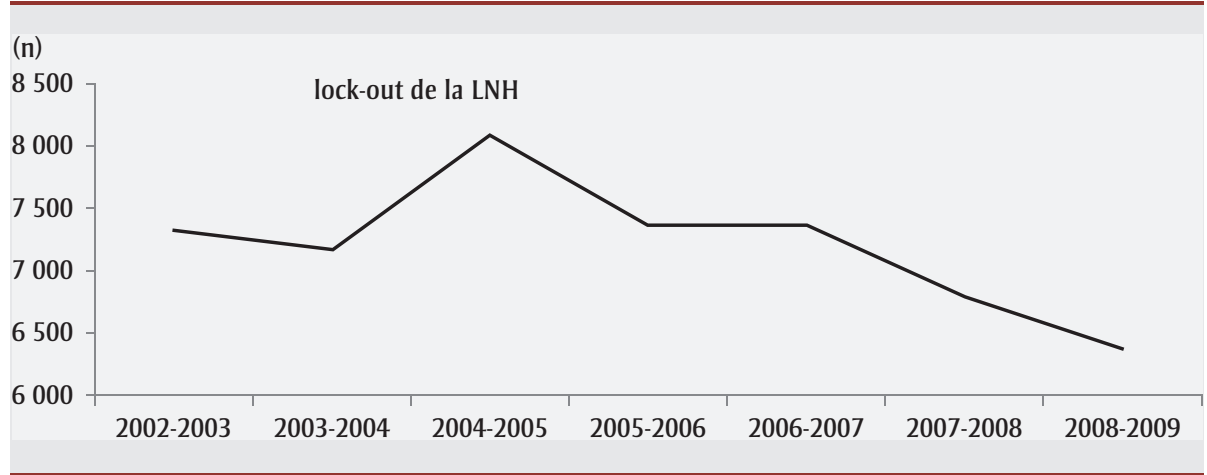

Sources : www.theahl.com, www.Inah.com

Abréviation : LNH, Ligue nationale de hockey

\section{Limites}

Nous reconnaissons l'existence de plusieurs limites. Tout d'abord, les données du SCHIRPT ne constituent qu'une fraction de toutes les blessures subies au Canada, laquelle ne peut être considérée comme un échantillon véritable de ces blessures. Les blessures traitées dans les services d'urgence des hôpitaux pédiatriques ne correspondent pas nécessairement aux données du dénominateur, qui concernent les joueurs inscrits. Nous reconnaissons donc que les taux que nous utilisons ne sont pas de véritables taux, car les données des numérateurs et des dénominateurs proviennent de populations quelque peu différentes. Toutefois, ce sont les comparaisons relatives qui nous intéressent, et il n'existe aucune raison de croire que la relation a changé au cours de la période de l'étude.

Une seconde limite est que, bien souvent, les rapports du SCHIRPT ne comportent pas suffisamment de détails pour permettre de nous assurer qu'une blessure a bien été causée par un acte agressif ou illégal; par ailleurs, certaines données sont parfois manquantes. Toutefois, le codage des dossiers est centralisé et est effectué par des préposés qualifiés, et l'information sur la nature de la blessure et le niveau de traitement est généralement uniforme au fil du temps. Encore une fois, sauf s'il existe une raison de supposer un changement de ces variables avec le temps, nos comparaisons restent justifiées.
Troisièmement, nous n'avons pas tenté de vérifier que tous les sujets de notre étude avaient bel et bien regardé des matchs de la LNH à la télévision entre 2002 et 2008. Toutefois, la Canadian Broadcasting Corporation (CBC) a récemment annoncé que son émission Hockey Night in Canada occupait le premier rang des cotes d'écoute pour l'ensemble de ses émissions; elle estime que $78 \%$ des Canadiens de 25 à 54 ans regardent les matchs de la $\mathrm{LNH}^{43}$. Si nous appliquons la même proportion à notre groupe cible d'adolescents de sexe masculin de 11 à 17 ans et vivant au Canada, tout en notant que les matchs de hockey de la LNH ont également été diffusés par d'autres chaînes de télévision que la $\mathrm{CBC}$, nous pouvons raisonnablement supposer qu'au moins un million de garçons de ce groupe d'âge regardent régulièrement les matchs de la LNH. Compte tenu de la place importante occupée par le hockey dans la culture canadienne, il serait surprenant que la des équipes locales ne soient pas également regardés. De plus, nous pensons raisonnable de présumer que, sauf pendant la saison du lock-out, durant laquelle il n'y avait aucun match à voir, la proportion de jeunes spectateurs est restée la même durant toute la période de l'étude.

Enfin, même si nous ne pouvons affirmer avec certitude que les jeunes hockeyeurs ont contribué à l'augmentation du nombre de spectateurs des matchs des ligues de majorité des matchs mettant en présence hockey mineur professionnel durant le lock-out, il semble raisonnable de supposer que cela a été le cas. Toutefois, bien que le nombre de spectateurs ait connu une hausse significative, même en incluant des enfants et des adolescents, nous sommes encore loin du nombre d'enfants et d'adolescents qui regardent le hockey à la télévision.

Il convient de souligner que les données présentées dans le tableau 1 laissent entrevoir un faible déclin de ces blessures avec le temps, même si les différences ne sont pas significatives selon le test de tendance de Jonckheere $(p=0,099)$. Si elle s'avérait, cette évolution pourrait être le résultat soit du succès de différentes initiatives de prévention, soit d'une réduction de la propension à se rendre aux urgences en cas de blessure.

\section{Conclusion}

Malgré une hypothèse raisonnable, nous n'avons pas réussi à démontrer que le fait de ne pas voir les scènes violentes, si caractéristiques du hockey professionnel, avait un effet bénéfique sur le comportement des jeunes joueurs. Plus précisément, nous n'avons observé aucune différence significative dans les taux de blessures pendant une année où les joueurs de hockey professionnels étaient en lock-out et où il n'y a donc pas eu de retransmission télévisée de leurs matchs. Toutefois, l'effet pourrait avoir été en partie masqué par le fait d'assister, en revanche, à des matchs des ligues de hockey junior, où la violence est encore plus présente.

\section{Remerciements}

Nous remercions les administrateurs du SCHIRPT de nous avoir permis d'utiliser les données de leurs hôpitaux, et sommes redevables à Steven McFaull et à Robin Skinner, de Santé Canada, d'avoir mis les données du SCHIRPT à notre disposition.

\section{Références}

1. Maccoby EE. Television : its impact on school children. Public Opin Quart. 1951;15(3):421-4. 
2. Bandura A, Walters RH. Social learning and personality development. New York : Holt, Rinehart and Winston; 1963.

3. Berkowitz L. Aggression : a social psychological analysis. New York : McGraw-Hill; 1962.

4. Rothenberg MB. Effect of television violence on children and youth. JAMA. 1975;234(10):1043-6.

5. Ferguson CJ. Media violence : miscast causality. Am Psychol. 2002;57(6-7):4467.

6. Ybarra ML. Linkages between depressive symptomatology and Internet harassment among young regular Internet users. Cyberpsychol Behav. 2004;7(2):247-57.

7. Villani S. Impact of media on children and adolescents : a 10-year review of the research. J Am Acad Child Psychiatry. 2001;40(4):392-401.

8. Bar-on ME. The effects of television on child health : implications and recommendations. Arch Dis Child. 2000;83(4):289292.

9. Browne KD, Hamilton-Giachritsis C. The influence of violent media on children and adolescents : a public-health approach. Lancet. 2005;365(9460):702-10.

10. Hopf WH, Huber GL, Weiß RH. Media violence and youth violence : a 2-year longitudinal study. J Media Psychol. 2008;20(3):79-96.

11. Huesmann LR, Taylor LD. The role of media violence in violent behavior. Annu Rev Publ Health. 2006;27:393-415.

12. McIntyre JJ; American Psychological Association. Testimony before the United States Senate Committee on Commerce, Science, and Transportation on the impact of media violence on children [Internet]. 2007 [consultation le 7 sept. 2011]. Consultable en ligne à la page : http:// www.apa.org/about/gr/pi/advocacy/2007 /mcintyre-media.aspx

13. Council on Communications and Media. From the American Academy of Pediatrics : policy statement-media violence. Pediatrics. 2009;124(5):1495-503.
14. Benson BW, Meeuwisse WH. Ice hockey injuries. Med Sport Sci. 2005;49:86-119.

15. Boden BP, Jarvis CG. Spinal injuries in sports. Neurol Clin. 2008;26(1):63-78.

16. Singer, DM. Hockey Fights [Internet]. [consultation le 7 sept. 2011]. Consultable en ligne à la page : http://www.hockeyfights .com

17. TSN. NHL injuries [Internet]. [consultation le 7 sept. 2011]. Consultable en ligne à la page : http://www.tsn.ca/nhl/injuries/

18. NHL fights [Internet]. [consultation le 7 sept. 2011]. Consultable en ligne à la page : http://dropyourgloves.com/Fights/ LeagueFights.aspx? League $=1$

19. IIHF. IIHF Rule Book, 2010-2014 [Internet] Zurich $(\mathrm{CH})$ : International Ice Hockey Federation; 2010 [consultation le 7 sept. 2011]. Consultable en ligne à la page : http://www.iihf.com/iihf-home/sport/iihfrule-book.html

20. Associated Press. NHL GMs form committee to study headshots [Internet]. Ottawa (Ont.) : The National Hockey League; 11 nov. 2009 [consultation le 7 sept. 2011]. Consultable en ligne à la page : http:// www.nhl.com/ice/news.htm?id = 505769

21. Duhatschek E. Should visors be mandatory in the NHL? [Internet]. Toronto (Ont.) : The Globe and Mail; 22 mars 2011 [consultation le 7 janv. 2012]. Consultable en ligne à la page : http://m.theglobeandmail.com /sports/hockey/globe-on-hockey/shouldvisors-be-mandatory-in-the-nhl/article1951461/?service $=$ mobile.

22. Anderson D. Sports of the times; the great visor debate in hockey [Internet]. New York : The New York Times; 22 mars 2000 [consultation le 7 janv. 2012]; Consultable en ligne à la page : http://www .nytimes.com/2000/03/22/sports/sports-ofthe-times-the-great-visor-debate-in-hockey .html.

23. Cusimano MD. Canadian minor hockey participants' knowledge about concussion. Can J Neurol Sci. 2009;36(3):315-20.

24. Gee CJ, Leith LM. Aggressive behavior in professional ice hockey : a cross-cultural comparison of North American and European born NHL players. Psychol Sport Exerc. 2007;8(4):567-83.
25. Nash JE, Lerner E. Learning from the pros : violence in youth hockey. Youth Soc. 1981;13(2):229-44.

26. Kerr JH. Book review : Aggression in the sports world : a social psychological perspective by Gordon W. Russell. International Journal of Sport Management and Marketing. 2009;5(4):477-8.

27. Smith MD. Violence and injuries in ice hockey. Clin J Sport Med. 1991;1(2):104-9.

28. Smith MD. Violence and sport. Toronto (Ont.) : Butterworths; 1983.

29. Smith MD. Significant others' influence on the assaultive behavior of young hockey players. Int Rev Sociol Sport. 1974;9(3):4558.

30. Pascall B, White S. Eliminating violence in hockey : a report. [Victoria] (BC) : British Columbia, Ministry of Small Business, Tourism and Culture; 2000 May.

31. Hockey Canada. Articles, statuts, règlements, historique. Tels qu'adoptés à Ottawa, le 4 décembre 1914 et amendés jusqu'en juin 2009. En vigueur à compter de la saison 2009-2010 [Internet]. [Ottawa] : Hockey Canada; 2009 [consultation le 7 sept. 2011]. Consultable en ligne à la page : http://www.hockeycanada.ca/index.php/ci _id/66601/la_id/2.htm

32. Système canadien hospitalier d'information et de recherche en prévention des traumatismes (SCHIRPT) [Internet]. Ottawa (Ont.) : Agence de la santé publique du Canada; 2011 [consultation le 7 sept. 2011]. Consultable en ligne à la page : http:// www.phac-aspc.gc.ca/injury-bles/chirpp /index-fra.php

33. Mackenzie SG, Pless IB. CHIRPP : Canada's principal injury surveillance program. Inj Prev. 1999;5(3):208-13.

34. Zhang JJ, Lam ETC, Connaughton EP, Bennett G, Pease DG, Pham UL et collab. Variables affecting spectator enjoyment of minor league hockey games. International Journal of Sport Management. 2004;5(2):126. 
35. Andrew DPS, Koo G-Y, Hardin R, Greenwell TC. Analysing motives of minor league hockey fans : the introduction of violence as a spectator motive. International Journal of Sport Management and Marketing. 2009;5(1-2):73-89.

36. Andrijiw AM. Life after hockey : an examination of athletic career transition and the National Hockey League's career transition program. Thesis (M.A.). St. Catharines (Ont.) : Faculty of Applied Health Sciences, Brock University; 2010.

37. Conacher B. As the puck turns : a personal journey through the world of hockey. Mississauga (Ont.) : J. Wiley \& Sons Canada; 2007.

38. Robidoux M, Trudel P. Hockey Canada and the bodychecking debate in minor hockey. In: David Whitson D, Gruneau R, (dir.). Artificial ice : hockey, culture, and commerce. Toronto (Ont.) : University of Toronto Press; 2006:101-22.

39. Reid SR, Losek JD. Factors associated with significant injuries in youth ice hockey players. Pediatr Emerg Care. 1999; 15(5):310-3.

40. Smith M. From professional to youth hockey violence : the role of the mass media. Dans: Gammon MAB, (dir.), Violence in Canada. Toronto (Ont.) : Methuen; 1978:269-81.

41. Latour G. La LNAH à l'heure des choix [Internet]. Hockey Zone Plus; 29 mars 2006 [consultation le 7 sept. 2011]. Consultable en ligne à la page : http://www .hockeyzoneplus.com/semipro/sp129_f.htm

42. American Hockey League. AHL attendance surpasses 7 million [Internet]. 22 mai 2005 [consultation le 7 sept. 2011]. Consultable en ligne à la page : http://theahl.com/ahlattendance-surpasses-7-million-p136423

43. \# 1 NHL broadcast in Canada! CBC report, 2012 [consultation le 28 mars 2012]. PDF (247 Ko) téléchargeable à partir du lien : http://www.cbc.ca/revenuegroup/images /HNIC\% 20-\%20Fall\% 202011.pdf 